\title{
Propiedades psicométricas del cuestionario de Bienestar Sexual Subjetivo en jóvenes y adultos chilenos
}

\section{Psychometric properties of the sexual subjetive well-being scale in young and adults chilean.}

\author{
Daniela Contreras \\ Daniela Jaime \\ Sebastián Lillo \\ $\mathrm{y}$ \\ Pablo Vera-Villarroel \\ Universidad de Santiago de Chile, USACH*
}

Rec (03 de Marzo de 2017) Acept (15 de Junio de 2017)

\begin{abstract}
Resumen
El presente estudio evalúa las propiedades psicométricas del cuestionario de bienestar sexual subjetivo en jóvenes y adultos chilenos residentes en Santiago de Chile. Se aplicó la versión de la escala traducida al español a una muestra de 661 jóvenes y adultos con edades comprendidas entre 18 y 54 años que no reportaron alguna enfermedad mental o física. El $49 \%$ de la muestra estuvo conformada por mujeres y el $51 \%$ por hombres. Las propiedades psicométricas y confiabilidad fueron evaluadas mediante análisis factorial exploratorio y alpha de Cronbach. La estructura factorial óptima del cuestionario fue la de un factor con 4 ítems y presenta una confiabilidad adecuada $(\alpha=.73)$, aunque el ítem 4 presenta cargas factoriales bajas. Los resultados indican que la versión chilena del cuestionario de bienestar sexual subjetivo es un instrumento autoadministrado válido y confiable para evaluar el bienestar en el ámbito sexual de mujeres y hombres sin reporte de enfermedad física o mental.

Palabras Claves: Bienestar sexual subjetivo, Propiedades psicométricas, Cuestionario breve.
\end{abstract}

\begin{abstract}
The present study evaluates the psychometric properties of subjective sexual well-being questionnaire in young and adult Chileans who residing in Santiago. The version of the scale translated into Spanish, was applied to a sample of 661 young and adults people between the ages of 18 and 54 who did not report any mental or physical illness. $49 \%$ of the sample was women and $51 \%$ of men. The psychometric properties and reliability were evaluated by exploratory factorial analysis and Cronbach's alpha. The optimal factorial structure of the questionnaire was one factor with 4 items and had adequate reliability $(\alpha=.73)$, although item 4 had low factor loadings. The results indicate that the version of the subjective sexual well-being questionnaire is a valid and reliable self-administered instrument to evaluate the sexual well-being of women and men without reports of physical or mental illness.

Keywords: Subjective sexual wellbeing, Psychometrics properties, Brief questionnaire.
\end{abstract}

\footnotetext{
Correspondencia: Daniela Contreras; daniela.contreras@usach.cl y Pablo Vera Villarroel, pablo.vera@usach.cl. Centro de Innovación y Tecnologías de la Información para Aplicaciones Sociales, CITIAPS, Universidad de Santiago de Chile. Avenida Ecuador 3650.

Nota: Esta investigación contó con el apoyo del proyecto FONDECYT No 1110520, Universidad de Santiago de Chile, USACH
} 


\section{Introducción}

El sexo es un aspecto fundamental en la sexualidad, el desarrollo y la vida de los seres humanos (Laumann et al., 2006; Programa de las Naciones Unidas para el Desarrollo [PNUD], 2012; World Health Organization [WHO], 2010).

En las últimas décadas, el interés en comprender la sexualidad ha ido en aumento, sin embargo, la investigación científica y el debate socio-político ha estado principalmente centrado en los problemas y disfunciones sexuales de la población, para lo cual se han desarrollado diversos sistemas de evaluación y programas de tratamiento (Abdolmanafi et al., 2015; Bois, Bergeron, Rosen, Mayrand, Brassard \& Sadikaj, 2016; Burria, Radwana \& Bodenmanna, 2015; Dennerstein, Guthrie, Hayes, DeRogatis, \& Lehert, 2008; Granados \& Sierra, 2016; Heiman, Long, Smith, Fisher, Sand \& Rosen, 2011; Kucur Suna et al., 2016; Rosen et al., 2009; Velten, Scholten, Graham \& Margraf, 2017; Vera-Villarroel, Valtierra \& Contreras, 2016; Willi, \& Burri, 2015).

Con el fin de comprender aquellos aspectos del sexo que contribuyen a la salud sexual y al bienestar del ser humano desde una perspectiva no patológica, se ha desarrollado un variado e incipiente cuerpo investigativo, principalmente en torno al concepto de satisfacción sexual (Bancroft, Long \& McCabe, 2011; Bridges, Lease, \& Ellison, 2004; Rosen \& Bachmann, 2008; Stulhofer, Busko, \& Brouillard, 2010; Sanchez-Fuentes, Salinas, \& Sierra, 2016). La satisfacción sexual se ha asociado a la evaluación que el individuo hace de los aspectos del sexo que tienen que ver con el ámbito personal (los aspectos emocionales y físicos del sexo), y con el ámbito interpersonal (comunicación sexual), así como la evaluación del aspecto subjetivo de satisfacción física alcanzada por los individuos en las diferentes etapas de la respuesta sexual (frecuencia, duración, cambios fisiológicos, etc.) (Pascoal, Narciso \& Pereira, 2014).

Siguiendo la línea de investigación en satisfacción sexual, recientemente ha surgido el concepto de bienestar sexual subjetivo. Este nuevo enfoque se centra en el papel positivo de las variables psicológicas para lograr una vida sexual más satisfactoria, apoyado en los postulados de la psicología positiva y más concretamente en los estudios de bienestar subjetivo (Laumann et al., 2006; Park, Peterson \& Sun, 2013; Silva et al. 2016; Rosen et al., 2009; Træen, \& Schaller, 2010).

Autores como Öberg, Fugl-Meyer, y Fugl-Meyer (2002) y Laumann et al. (2006) han descrito el bienestar sexual subjetivo como la evaluación cognitiva y emocional de un individuo, que incorpora la calidad percibida de su propia sexualidad y las relaciones sexuales. El bienestar sexual subjetivo sería una evaluación más amplia y global de la experiencia sexual, al conceptualizarla no sólo desde la función sexual.

Las investigaciones en torno al bienestar sexual subjetivo son escasas y presentan diferencias conceptuales y metodológicas en su abordaje (Pascoal et al., 2014). Algunas de ellas utilizan indiferenciadamente el término satisfacción sexual y bienestar sexual subjetivo para evaluar este último constructo (Bancroft et al., 2011; Graf \& Patrick, 2014; Hooghe, 2012; Kedde, van de Wiel, Weijmar Schultz \& Wijsen, 2013; Stephenson, \& Meston, 2010a; ZimmerGembeck \& French, 2016), e inclusive otras pretenden evaluar satisfacción sexual, pero su definición conceptual se aproxima más al enfoque de bienestar sexual subjetivo (Stulhofer et al., 2010). Esta falta de claridad en su definición conceptual limita el análisis del constructo, su validación y diferenciación de otros conceptos utilizados tradicionalmente en el campo de la sexualidad (Pascoal et al., 2014).

Entre aquellas investigaciones que han desarrollado cuestionarios y preguntas del bienestar sexual subjetivo se encuentra el estudio de Hooghe (2012), quien evalúa la confiabilidad y validez de una escala tradicional de bienestar subjetivo con incorporación de una pregunta general que apunta al bienestar sexual. Los resultados de este estudio sugieren el potencial de incluir una pregunta de bienestar sexual en las evaluaciones de bienestar subjetivo, debido al aumento de la confiabilidad, desde un alfa de Cronbach de .70 a .75 , luego de incluir la pregunta sobre bienestar sexual.

Rosen et al. (2009) desarrollaron y validaron un cuestionario de bienestar sexual en población femenina que constaba de 17 preguntas. Los resultados dan cuenta de un instrumento multidimensional fiable, con un alto grado de coherencia interna para los 4 dominios del instrumento y fiabilidad test-retest alta en las mujeres de diferentes edades.

Por su parte Laumann et al. (2006) desarrollaron un cuestionario para evaluar el bienestar sexual subjetivo en personas mayores de 40 años. El cuestionario tiene cuatro preguntas que representan las cuatro dimensiones del bienestar sexual subjetivo: aspectos emocionales en las relaciones sexuales, aspectos físicos en las relaciones sexuales, funcionamiento sexual e importancia de la sexualidad en la vida en general de las personas. Estas preguntas fueron validadas con una muestra de 27.514 personas pertenecientes a 29 países de diversos continentes. Los resultados de la evaluación de la estructura factorial muestran un factor dominante en los diferentes países. La satisfacción física y emocional mostraron una correlación más fuerte $(r=.80)$ en relación con los otros dos aspectos evaluados. Junto con lo anterior, el estudio de Laumann et al. (2006), evalúa la asociación entre 
bienestar sexual subjetivo y variables del comportamiento sexual, reportándose un asociación directa significativa entre la frecuencia sexual, número de parejas y juegos sexuales.

Otras investigaciones en esta línea, son las de Bancroft, Long y McCabe (2011), quienes evalúan este constructo en mujeres mediante dos preguntas que apuntan a la satisfacción con la relación sexual actual y con su propia sexualidad.

Por su parte, Træen y Schaller (2010) realizaron una investigación en una muestra de adultos heterosexuales noruegos (18 y 67 años). Encontraron que el bienestar sexual subjetivo decrece con la edad, y que existen diferencias entre hombres y mujeres. Las mujeres jóvenes están más satisfechas con su vida sexual que los hombres jóvenes, pero a medida que aumenta la edad esta relación se invierte.

En un estudio más reciente, Silva et al. (2016), estudiaron el bienestar sexual subjetivo asociado al funcionamiento sexual, la inteligencia emocional en portugueses adultos, encontrando diferencias entre hombres y mujeres en las tres variables estudiadas. Las mujeres poseen mayor inteligencia emocional que los hombres, sin embargo, los hombres presentaron mayores niveles de bienestar sexual subjetivo. Además encontraron asociaciones significativas, pero moderadas entre la inteligencia emocional y las variables sexuales.

Otras investigaciones en el ámbito de la sexualidad también han reportado una relación significativa entre el comportamiento sexual y conceptos como satisfacción sexual, calidad de vida, bienestar y felicidad (Carrobles et al., 2011; Cheng, \& Smyth, 2015; Contreras, Lillo \& Vera-Villarroel, 2016; Heiman et al., 2011; Holmberg, Blair \& Phillips, 2010; Mastro \& Zimmer-Gembeck, 2015; Lee, Vanhoutte, Nazroo \& Pendleton, 2016; Træen, \& Schaller, 2010; Woloski-Wruble, Oliel, Leefsma \& Hochner-Celnikier, 2010).

En la población hispano hablante, la mayoría de los estudios recientes se han centrado en el funcionamiento sexual y sus disfunciones (Bravo, Meléndez, \& López, 2005; Carrobles et al., 2011; da Silva et al., 2012; Ribeiro, Nakamura, Scanavino, Torlonj \& Mattsr, 2011; SánchezFuentes et al., 2016; Vallejo-Medina \& Sierra, 2013), la asertividad sexual (Santos-Iglesias \& Sierra, 2010; SantosIglesias, Sierra \& Vallejo-Medina, 2013) o las fantasías sexuales (Sierra, Ortega, Martín-Ortiz \& Vera-Villarroel, 2004; Moyano \& Sierra, 2012).

Son escasos los estudios acerca del bienestar sexual subjetivo en latinoamérica, dentro de ellos se encuentra el realizado por Contreras, Lillo y Vera-Villarroel (2016), quienes desarrollaron y evaluaron un modelo predictivo de bienestar sexual subjetivo. En este estudio encontraron que variables asociadas al comportamiento sexual (frecuencia sexual, caricias y contacto sexual), así como la felicidad y la percepción de salud estaban asociadas al bienestar sexual subjetivo.

Considerando el bajo número de estudios en cultura latinoamericana respecto al bienestar sexual subjetivo y sus sistemas de evaluación, el presente estudio tuvo como objetivo evaluar la fiabilidad y validez de la medida psicométrica breve de bienestar sexual subjetivo de Laumann et al. (2006) en población chilena, dado que es una de las pocas medidas que se ha aplicado tanto a hombres como mujeres de distintas edades, naciones y culturas. Específicamente, se espera poner a prueba la estructura factorial del instrumento, evaluar su fiabilidad y obtener indicadores de validez, comparando sexo y edad.

\section{Método}

\section{Participantes}

Un total de 661 personas respondieron el cuestionario, las cuales cumplían con los criterios de inclusión: ser mayor de edad y el haber tenido al menos una relación sexual coital en los últimos doce meses. Además, las personas eran alfabetas, autovalentes, residentes en la ciudad de Santiago y no presentaban problemas físicos o mentales que limitaran la autoaplicación del instrumento, con ausencia de un cuadro de salud mental y/o enfermedades físicas crónicas en los últimos cinco años.

Los participantes tenían entre 18 y 54 años de edad. La edad promedio de los participantes fue 31 años $(M=30.65$ $D T=9.78$ años $)$, el $54.31 \%(n=359)$ con edades entre 18 y 29 años, mientras que el $46 \%(n=302)$, con edades entre 30 y 54 años.

E1 51\% de la muestra estuvo conformada por hombres y el $49 \%$ por mujeres. Los participantes presentaron un nivel educativo elevado; $57 \%$ indicó que su último nivel educativo cursado fue Enseñanza Superior; sólo el $5 \%$ indicó estar cursando o haber terminado la Enseñanza Básica. Al momento de contestar el cuestionario, el $63 \%$ de los participantes declararon estar soltero (con y sin compromiso), el $12 \%$ se encontraba conviviendo, y el 19\% indicó estar casado/a. El $4 \%$ indicó estar divorciado o separado, mientras que cerca del $1 \%$ indicaron ser viudos/as (ver tabla 1 ). 
Tabla 1. Características Sociodemográficas de la muestra

\begin{tabular}{lcc}
\hline Edad & $\mathrm{n}$ & $\%$ \\
$18-29$ & 359 & $54.3 \%$ \\
$30-54$ & 302 & $45.7 \%$ \\
\hline Sexo & & \\
Hombre & 340 & $51.4 \%$ \\
Mujer & 321 & $48.6 \%$ \\
\hline Nivel Educacional & & \\
Básica & 33 & $5.0 \%$ \\
Media & 251 & $38.0 \%$ \\
Superior & 377 & $57.0 \%$ \\
\hline Situación Sentimental & & \\
Soltero/a Sin compromiso & 235 & $35.6 \%$ \\
Soltero/a con compromiso & 184 & $27.8 \%$ \\
Conviviente & 81 & $12.3 \%$ \\
Casado/a & 125 & $18.9 \%$ \\
Divorciado/Separado & 27 & $4.1 \%$ \\
Viudo & 9 & $1.4 \%$ \\
\hline
\end{tabular}

\section{Diseño y procedimiento}

Se usó un diseño transversal, con un muestreo de tipo intencional, en el cual los datos fueron recolectados a través de un cuestionario de autorreporte de lápiz y papel aplicado por encuestadores cara a cara en distintas instituciones y lugares de trabajo (universidades, escuelas, empresas, etc.) en la Región Metropolitana de Chile.

Los objetivos de la investigación, procedimientos, y consentimiento informado fueron evaluados previamente por el comité de ética institucional de la Universidad de Santiago y el comité de la institución patrocinadora.

La administración del cuestionario siguió las normas éticas internacionales indicadas para este tipo de investigación, por lo tanto, las personas que aceptaron responder a la prueba firmaron un consentimiento informado, que también tuvo que ser firmado por los entrevistadores, con copia a los participantes.

En una primera etapa, las preguntas para medir el bienestar subjetivo y el comportamiento sexual fueron traducidas del inglés al español y luego del español al inglés por dos traductores independientes. Ambas versiones fueron comparadas y discutidas por parte de los investigadores. La versión final de la prueba quedó escrita en español comprensible para la población chilena. El procedimiento siguió las recomendaciones de Brislin (1970) y Merenda (2006).

Posteriormente, se realizó la aplicación de una prueba piloto a un grupo de doce personas que respondieron el cuestionario, y se solicitó la opinión de dos psicólogos expertos, externos a la investigación, lo que permitió mejorar la claridad conceptual y comprensión de las preguntas.

En una segunda etapa, se realizó la aplicación del cuestionario, contando para ello con encuestadores profesionales entrenados, quienes solicitaron a las personas, cara a cara y de forma voluntaria, responder el instrumento de evaluación en lápiz y papel. Ninguno de los participantes recibió retribución económica por contestar la encuesta.

\section{Instrumentos y medidas}

Información sociodemográfica. Los participantes respondieron a una serie de preguntas que se refieren a los datos sociodemográficos de la población estudiada (edad, estado civil y nivel educacional) y su salud física y mental actual (criterio de exclusión del estudio).

Bienestar sexual subjetivo. Para medir el bienestar sexual subjetivo se utilizó el instrumento desarrollado por Laumann et al. (2006). La escala consta de 4 preguntas que miden la satisfacción con distintos aspectos de la actividad y vida sexual. La primera pregunta evalúa satisfacción física en las relaciones sexuales de pareja: "Durante los últimos 12 meses, ¿cuán satisfactorias fisicamente han sido sus relaciones sexuales de pareja?"; la segunda se dirige a la satisfacción emocional en las relaciones sexuales: "Durante los últimos 12 meses, ¿cuán satisfecho/a emocionalmente ha estado en sus relaciones sexuales de pareja? Ambas preguntas se responden en una escala Likert de 5 puntos, que va desde "totalmente insatisfecho" a "totalmente satisfecho". Una tercera pregunta alude a la satisfacción con la función sexual: "Si usted tuviera que pasar el resto de su vida con su funcionamiento (función/salud) sexual actual ¿cómo se sentiría respecto a esto?; se responde en una escala Likert de 5 puntos, desde "muy insatisfecho" a "muy satisfecho". Por último, la cuarta pregunta evalúa la importancia del sexo: “¿Cuán importante en general diría usted es el sexo en su vida?; las opciones de respuesta van desde «nada importante" a "muy importante" en una escala Likert de 5 puntos.

Indicadores del comportamiento sexual. Para el análisis de validez predictiva del instrumento en estudio, se evaluaron indicadores del comportamiento sexual que habían mostrado relación con el bienestar sexual en estudios anteriores (Laumann et al., 2006, Heiman et al., 2011). Para ello se incluyeron cuatro preguntas que evalúan las prácticas sexuales de las personas, las cuales forman parte del International Survey of Relationships creado por Heiman y et al. (2011) a partir de diversos cuestionarios que evaluaban aspectos de la conducta sexual. Estas preguntas abordan las áreas 
de intimidad física e historia sexual. Dos de las preguntas miden la frecuencia de las relaciones sexuales en los últimos 4 meses y en los últimos 12 meses. Una de las preguntas aborda la cantidad de parejas sexuales en los últimos 12 meses. Finalmente, se les pregunta a los participantes por el tiempo promedio que dedica a los juegos previos a la relación sexual.

\section{Resultados}

\section{Estrategia Analítica}

Las propiedades psicométricas del cuestionario de Bienestar Sexual Subjetivo (Laumann et al., 2006) en población chilena, fueron evaluadas de la siguiente forma:

Los análisis de datos cuantitativos descriptivos tanto de la muestra como del cuestionario fueron realizados con el software R Versión 3.4.0 (R Development Core Team, 2017) usando el package $P$ sych (Revelle, 2017) y el package car (Fox \& Weisberg, 2011).

El análisis de factorial exploratorio para variables categóricas ordinales, con matriz de correlaciones policóricas y con el estimador robusto Weighted Least Squares (WLSMV) se realizó con el Software Mplus v. 7.4 (Muthén \& Muthén, 1998-2015).

Por otro lado, la consistencia interna se evaluó mediante el alfa de Cronbach. Los valores iguales o superiores a $\alpha=$ .70 fueron considerados satisfactorios. En este análisis se llevaron a cabo comparaciones de los resultados por sexo (Hombre y Mujer) y edad (18-29 y 30-54).

Adicionalmente, y como apoyo a la evidencia de validez de la escala, se evaluó empíricamente su utilidad a través de una comparación de medias por sexo y edad, y también un análisis de regresión múltiple.

\section{Análisis Factorial}

En primer lugar, se analizó si la matriz de correlaciones de los 4 ítems de la escala era apropiada para realizar un análisis factorial. La prueba de esfericidad de Bartlett $(\chi 2=776.8942$; $d f=6 ; p<.001)$ indicó que los ítems no eran independientes. Junto con ello, el coeficiente de Kaiser-Meyer-Olkin (KMO $=.71$ ), indica que las correlaciones entre parejas de ítems podían ser explicadas por los ítems restantes.

La estructura factorial de la escala de bienestar sexual subjetivo fue evaluada través de un análisis factorial exploratorio para variables categóricas con correlaciones policóricas y rotación Geomin. El estimador utilizado fue WLSMV, debido a que los ítems no se distribuyen normalmente, pues presentan asimetrías negativas (Véase tabla 2).

Se obtuvo una solución de 1 factor. Los ítems de satisfacción sexual física y emocional, junto con el de función sexual mostraron cargas factoriales sobre .70, mientras que el ítem de importancia del sexo en la vida mostró una carga factorial de .35. Tomando en cuenta estos resultados, idealmente se debería eliminar este último ítem, sin embargo, dada la poca cantidad de ítems, al ser eliminado no se pueden calcular los índices de ajuste porque no quedan parámetros libres para hacerlo. En este sentido, los índices de ajuste de la escala con 4 ítems resultan muy buenos de acuerdo a los criterios de $\mathrm{Hu}$ y Bentler (1999): El indíce $\chi 2 / \mathrm{df}=3.25$, se encuentra debajo del criterio de corte 3.8. Lo mismo ocurre con el indicador Root Mean Square Error of Approximation (RMSEA= .058) que se encuentra por debajo de .06, en donde y el Standardized Root Mean Square Residual (SRMR= .021) en valores menores a .08. Los parámetros Comparative Fit Index (CFI) y el Tucker-Lewis Index (TLI), también se encuentran en valores óptimos, por sobre .95 .

\section{Análisis de items y consistencia interna}

Como muestra la tabla 3, el valor del alfa de Cronbach total fue de .73, mostrando un comportamiento valores menores en hombres y que en mujeres $(\alpha=.68$ y $\alpha=.77$ respectivamente). Dado el bajo número de ítems de la escala, estos valores son suficientes para evidenciar una adecuada consistencia interna entre las puntuaciones obtenidas.

En cuanto a la comparación por edad, los valores de alfa de Cronbach aparecen más elevados en el grupo de menor edad (18 a 29 años), con un $\alpha=.68$, en comparación al grupo mayor $(\alpha=.77)$ aunque ambos en un nivel adecuado.

Tabla 2. Distribución de respuesta por item (1 a 5)

\begin{tabular}{lccccccc}
\hline \multicolumn{1}{c}{ Ítem } & \%Resp 1 & \%Resp 2 & \%Resp 3 & \%Resp 4 & \%Resp 5 & Asimetría & Curtósis \\
\hline 1. Satisfacción física & 4.1 & 4.2 & 17.5 & 41.6 & 32.5 & -1.06 & 0.92 \\
2. Satisfacción emocional & 3.2 & 5.6 & 23.6 & 40.4 & 27.2 & -0.77 & 0.35 \\
3. Función Sexual & 7.1 & 6.4 & 15.7 & 43.3 & 27.5 & -1 & 0.39 \\
4. Importancia del sexo & 0.9 & 6.1 & 5.7 & 53.1 & 34.2 & -1.24 & 1.87 \\
\hline
\end{tabular}


Tabla 3. Análisis Factorial Exploratorio de la Escala de bienestar sexual subjetivo

\begin{tabular}{lc}
\hline \multicolumn{1}{c}{ Ítem } & Cargas Factoriales \\
\hline 1. Satisfacción física & $.865^{*}$ \\
2. Satisfacción emocional & $.907^{*}$ \\
3. Función sexual & $.732^{*}$ \\
4. .Importancia del sexo & $.352^{*}$ \\
\hline
\end{tabular}

$*_{p}<.05$

Tabla 4. Confiabilidad (a) si el item es eliminado, por sexo, edad y muestra total.

\begin{tabular}{lccccc}
\hline & Total & \multicolumn{2}{c}{ Sexo } & \multicolumn{2}{c}{ Edad } \\
\hline \multicolumn{1}{c}{ Ítem } & $\mathrm{n}=661$ & Hombres $(\mathrm{n}=340)$ & Mujeres $(\mathrm{n}=321)$ & $18-29(\mathrm{n}=359)$ & $30-54(\mathrm{n}=302)$ \\
\hline 1 Satisfacción física & .58 & .52 & .65 & .52 & .67 \\
2 Satisfacción emocional & .57 & .51 & .65 & .51 & .65 \\
3 Función sexual & .64 & .6 & .68 & .67 & .79 \\
4 Importancia del sexo & .82 & .68 & .79 & .68 & .85 \\
\hline Bienestar sexual subjetivo & .73 & .78 & .78 \\
\hline
\end{tabular}

Tabla 5. Análisis de regresión múltiple de la escala de bienestar sexual subjetivo

\begin{tabular}{|c|c|c|c|c|}
\hline Ítem & $\mathrm{B}$ & Error típico & $\beta$ & $\mathrm{p}$ \\
\hline (Intercepto) & 2.589 & 0.108 & & $* * *$ \\
\hline 1. En las últimas 4 semanas ¿Cuántas veces ud. ha tenido relaciones sexuales? & 0.172 & 0.02 & 0.335 & $* * *$ \\
\hline 2. En los últimos 12 meses ¿Con que frecuencia promedio ha tenido relaciones sexuales? & 0.195 & 0.035 & 0.223 & $* * *$ \\
\hline 3. ¿Cuántas parejas sexuales ha tenido en los últimos 12 meses? & -0.022 & 0.011 & -0.07 & * \\
\hline $\begin{array}{l}\text { 4. ¿Qué cantidad de tiempo promedio dedica a los juegos sexuales previos a una } \\
\text { relación sexual? }\end{array}$ & 0.113 & 0.022 & 0.165 & $* * *$ \\
\hline
\end{tabular}

$* p<.05 ; * * * p<.001$

\section{Bienestar sexual subjetivo por sexo y por edad}

Se realizó una prueba t para comparar el promedio de bienestar sexual por sexo y por edad. Los resultados indican que no existen diferencias significativas en el bienestar sexual subjetivo por sexo $(t(645.78)=-0.234, p>.05)$, ni por edad $(t(634.68)=0.181, p>.05)$. En este sentido, hombres $(M=3.91, D T=0.72)$ y mujeres $(M=3.93, D T=0.78)$ presentan en promedio similares niveles de bienestar sexual. Lo mismo ocurre entre los jóvenes $(M=3.93, D T=0.74)$ y los adultos $(M=3.92, D T=0.76)$.

\section{Análisis de regresión lineal múltiple}

Se realizó un análisis de regresión lineal múltiple con el objetivo de contribuir al análisis de validez.
El análisis de regresión múltiple fue usado para probar si las variables asociadas a la intimidad física predicen significativamente el bienestar sexual subjetivo. Los resultados indican que las 4 variables predicen un $31,51 \%$ de la varianza del bienestar sexual $(R 2=.3151, F(4.656)=$ $76.89, p<.001)$.

La Tabla 5 muestra que todas la variables asociadas a la intimidad física son predictoras significativas $(p<.05)$ del Bienestar Sexual Subjetivo. Las personas que reportaron una mayor frecuencia promedio de relaciones sexuales en los últimos doce meses $(b=0.223, p<.01)$, en las últimas cuatro semanas $(b=0.335, p<.01)$, y cuantas veces ha sido tocado o acariciado por una pareja $(b=0.165, p<.01)$, mostraban mayores niveles de bienestar sexual subjetivo. Asimismo, la cantidad de parejas sexuales predice significativamente el bienestar sexual subjetivo $(b=-0.07, p<.01)$, aquellas 
que tenían menos parejas sexuales en los últimos 12 meses, tienen mayor bienestar sexual subjetivo.

\section{Discusión}

El presente estudio evaluó las propiedades psicométricas de la escala de cuatro ítems de Bienestar Sexual Subjetivo de Laumann et al. (2006) en población chilena adulta. Los resultados reportaron que el instrumento autoadministrado es confiable para la evaluación de ámbito sexual del bienestar, tanto en hombres como mujeres sin patología sexual, física o mental autorreportada.

Si se consideran los cuatro ítems medidos, la escala presenta una adecuada consistencia interna y fiablidad, con sólo un factor para las cuatro preguntas analizadas. Estos resultados apoyan lo encontrado por Laumann et al. (2006) en el desarrollo y validación del instrumento, en el cual se reporta un factor dominante en las diferentes culturas y países donde se midió el bienestar sexual subjetivo.

Por otro lado, el análisis de la consistencia interna del instrumento muestra valores aceptables $(\alpha=.73)$, considerando el bajo número de ítems de la escala. Sin embargo, el ítem referido a la importancia del sexo para la persona, resulta ser menos consistente, dado que presenta una carga factorial baja $(<.40)$ y al ser eliminado la escala de bienestar subjetivo sube su confiabilidad $(\alpha=.90)$. El mal funcionamiento de este ítem podría deberse a que este no está dirigido a evaluar la satisfacción con el aspecto específico de la relación o de la vida sexual, sino que es una evaluación más global de la sexualidad. En otras palabras, para una persona puede ser muy importante el sexo en la vida, pero no necesariamente estar subjetivamente satisfecho con las relaciones sexuales con su pareja a nivel físico o emocional, lo que impacta a nivel de bienestar sexual subjetivo.

Estos resultados coinciden con lo encontrado por Laumann et al. (2006), donde la satisfacción física y la emocional aparecen como los ítems con mayor correlación y carga factorial, reafirmando con esto la importancia de la valoración tanto física como emocional en la evaluación del bienestar sexual subjetivo, como un constructo más amplio que el de satisfacción, centrada mayormente en la experiencia y valoración sexual física.

El análisis predictivo utilizado mostró que las variables del comportamiento sexual, obtienen resultados similares a los entregados en estudios previos (Carrobles et al., 2011; Contreras et al., 2016; Heiman et al., 2011; Holmberg et al., 2010; Silva et al., 2016; Woloski-Wruble et al., 2010) presentando una relación significativa con el bienestar sexual subjetivo.

respecto al análisis por edad y sexo, los resultados indicaron la ausencia de diferencias en ambas áreas en la puntuación del bienestar sexual subjetivo. Estos datos van en la misma dirección de los expuesto por Hooghe (2012) y por Contreras et al. (2016) en el área de Bienestar Sexual Subjetivo, aunque muestran diferencias con estudios más tradicionales de la investigación en sexualidad, como es el caso de las disfunciones sexuales (Laumann, Gagnon, Michael \& Michaels, 1994; McMahon et al., 2008) y satisfacción sexual, donde sí se muestran diferencias por sexo (Holmberg et al., 2010; Meston \& Trapnell, 2005) y edad (Davison, Bell, LaChina, Holden \& Davis, 2009).

Las diferencias encontradas con otras investigaciones en el área de sexualidad, podrían estar relacionadas con el foco de la evaluación que tienen los estudios en disfunción y satisfacción sexual, donde se miden generalmente aspectos biológicos relacionados al funcionamiento sexual físico, los cuales se ven mayormente afectados por el aumento de los años de vida (Mella, Oyadanel, Vargas \& de Ugarte, 2015).

Particularmente, en la evaluación del bienestar sexual subjetivo, estudios como el de (Heiman et al., 2011; Rosen et al., 2009; Træen \& Schaller, 2010), han reportado diferencias al medir este tipo de bienestar en personas adultas y adultas mayores. Estas diferencias reportadas pueden ser explicadas por la utilización de otros sistemas de medición del Bienestar, distintos al utilizado en esta investigación. Adicionalmente, la mayoría de los estudios mencionados realizaron mediciones sólo a grupos de mujeres, a diferencia del presente estudio que analizó tanto a hombres como mujeres.

En conclusión, los datos entregados a partir de la validación en población adulta chilena de la escala de bienestar Sexual, se muestran coherentes con la definición conceptual de bienestar sexual subjetivo, el cual aparece como un concepto y valoración más amplia de la experiencia sexual, no sólo limitada a la respuesta o rendimiento sexual, al incorporar el componente cognitivo y la experiencia subjetiva de esta, donde sexo y edad no estarían influenciando de manera significativa dicha experiencia evaluada.

En términos de la aplicabilidad del constructo en estudio, los resultados encontrados en la investigación vienen a confirmar que se puede contar con un instrumento adecuado para evaluar este constructo, otorgando estos resultados, evidencia para un sistema de medición confiable del bienestar sexual subjetivo en población adulta chilena.

Dentro de las limitaciones de la investigación, se encuentra la falta de análisis comparativos con estudios similares, al 
no contar aún con evidencia especialmente en culturas de Sudamérica de este constructo, lo que reduce la factibilidad de generalizar los principales resultados encontrados a otros países latinoamericanos.

Con respecto a las limitaciones en relación con la muestra, en primer lugar, los participantes del presente estudio fueron sujetos que mostraron disponibilidad a participar, y que cumplían con una serie de criterios de inclusión dentro de los que se encontraba presentar actividad sexual coital en el último año, por lo que puede haber un sesgo de autoselección en los resultados.

De la misma forma, es importante continuar ampliando los estudios para validar la escala de bienestar sexual subjetivo con muestras aleatorias en distintas regiones de Chile más allá de solo la ciudad de Santiago, incluyendo a adultos mayores y otras variables que pudiesen enriquecer las investigaciones en el área.

\section{Referencias}

Abdolmanafi, A., Azadfallah, P., Fata, L., Roosta, M., Peixoto, M.M., \& Nobre, P. (2015). Sexual Dysfunctional Beliefs Questionnaire (SDBQ): Translation and Psychometric Properties of the Iranian Version. The Journal of Sexual Medicine, 12(8), 1820-1827.

Bancroft, J., Long, J.S., \& McCabe, J. (2011). Sexual Well-Being: A Comparison of U.S. Black and White Women in Heterosexual Relationships. Archives of Sexual Behavior, 40(4), 725-740.

Bois, K., Bergeron, S., Rosen, N., Mayrand, M-H, Brassard, A., \& Sadikaj, G. (2016). Intimacy, Sexual Satisfaction, and Sexual Distress in Vulvodynia Couples: An Observational Study. Health Psychology, 35(6), 531-540.

Bravo, C.S., Melendez, J.C., Ramirez, S.M., \& Lopez, M. (2005). Feminine and masculine sexual dysfunctions: gender comparison in a Mexico City sample. Salud Mental, 28(4), 74-80.

Bridges, S. K., Lease, S. H., \& Ellison, C. R. (2004). Predicting sexual satisfaction in women: Implications for counselor education and training. Journal of Counseling \& Development, 82, 158-166.

Brislin, R. W. (1970). Back-Translation for Cross-Cultural Research. Journal of Cross-Cultural Psychology, 1(3), 185-216.

Burria, A., Radwana, S., \& Bodenmanna, G. (2015). The Role of PartnerRelated Fascination in the Association between Sexual Functioning and Relationship Satisfaction. Journal of Sex \& Marital Therapy, 41(6), 672-679.

Carrobles, J., Gámez-Guadix, J., \& Almendros, C. (2011). Funcionamiento sexual, satisfacción sexual y bienestar psicológico y subjetivo en una muestra de mujeres españolas. Anales de Psicología, 27(1), 27-34.

Contreras, D., Lillo, S., \& Vera-Villarroel P. (2016). Subjective Sexual Well-Being in Chilean Adults: Evaluation of a Predictive Model. Journal of Sex \& Marital Therapy, 42(4), 338-352.

Cheng, Z., \& Smyth, R. (2015). Sex and happiness. Journal of Economic Behavior \& Organization, 112, 26-32.

da Silva, E.A., Figueiredo, R., Barboza, R., Lessa, T., Vieira, R., \& Damiao R. (2012). The Impact of Perceived Ejaculate Volume on Sexual Satisfaction and Quality of Life. Journal of Sexual Medicine, 9, 79.

Davison, S., Bell, R., LaChina, M., Holden, S., \& Davis, S. (2009).The relationship between self-reported sexual satisfaction and general wellbeing in women. Journal of Sexual Medicine, 6, 2690-2697.

Dennerstein, L., Guthrie, J., Hayes, R., DeRogatis, L., \& Lehert, P. (2008). Sexual function, dysfunction, and sexual distress in a prospective, population-based sample of mid-aged, Australian-born women. Journal of Sexual Medicine, 5(10), 2291-2299.

Fox, J. \& Weisberg, S. (2011). An $\{R\}$ Companion to Applied Regression, Second Edition. Thousand Oaks CA: Sage.

Graf, A., \& Patrick, J.H. (2014). The influence of sexual attitudes on midto late-life sexual well-being: age, not gender, as a salient factor. The International Journal of Aging and Human Development, 79 (1), 55-79.

Granados, M. R., \& Sierra, J. C. (2016). Excitación sexual: una revisión sobre su relación con las conductas sexuales de riesgo. Terapia Psicológica, 34, 59-70.

Heiman, J., Long, J.S., Smith, S., Fisher, W., Sand, M., \& Rosen, R. (2011). Sexual Satisfaction and Relationship Happiness in Midlife and Older Couples in Five Countries. Archives of Sexual Behavior, 40(4), 741-753.

Holmberg, D., Blair, K., \& Phillips, M. (2010). Women's sexual satisfaction as a predictor of well-being in same-sex versus mixed-sex relationships. Journal of Sex Research, 47(1), 1- 11.

Hooghe, M. (2012). Sexual well-being part of subjective well-being? An empirical analysis of Belgian (Flemish) survey data using an extended well-being scale. Journal of Sex Research, 49(2-3), 264-27.

Hu, L. T., \& Bentler, P. M. (1999). Cutoff criteria for fit indexes in covariance structure analysis: Conventional criteria versus new alternatives. Structural equation modeling: a multidisciplinary journal, 6(1), 1-55.

Kedde, H., van de Wiel, H.B., Weijmar Schultz, W.C., \& Wijsen, C. (2013). Subjective sexual well-being and sexual behavior in young women with breast cancer. Support Care Cancer, 21(7), 1993-2005.

Kucur Suna, K., Ilaya, G., Aysenurb, A., Hanc, G.K., Ulkub, U.E., Pasad, U., \& Fatmae, C. (2016). Effects of Infertility Etiology and Depression on Female Sexual Function. Journal of Sex \& Marital Therapy, 42(1), 27-35.

Laumann E. O., Gagnon J. H, Michael R. T. \& Michaels S. (1994). The social organization of sexuality: Sexual practices in the United States. Chicago: University of Chicago Press.

Laumann, E. O, Paik, A., Glasser, D., Kang, J.H., Wang, T., Levinson, B., Moreira, E., Nicolosi, A. \& Gingell, C. (2006). A cross-national study of subjective sexual well-being among older women and men: Findings from the global study of sexual attitudes and behaviors. Archives of Sexual Behavior, 35(2), 145-161.

Lee, D. M., Vanhoutte, B., Nazroo, J., \& Pendleton, N. (2016). Sexual health and positive subjective well-being in partnered older men and women. Journals of Gerontology Series B: Psychological Sciences and Social Sciences, 71(4), 698-710.

Mastro, S., \& Zimmer-Gembeck, M. (2015). Let's talk openly about sex: Sexual communication, self-esteem and efficacy as correlates of sexual well-being. European Journal of Developmental Psychology, 12(5), 579-598.

Mella, C., Oyanedel, J., Vargas, S., \& de Ugarte, N. (2015). Salud sexual en Chile: una aproximación descriptiva al comportamiento y la satisfacción sexual de los chilenos. Revista Chilena de Obstetricia y Ginecología, 80(4), 289-296.

Merenda, P. F. (2006). An overview of adapting educational and psychological assessment instruments: past and present. Psychological Reports, 99(2), 307-314.

Meston, C., \& Trapnell, P. (2005). Development and Validation of a FiveFactor Sexual Satisfaction and Distress Scale for Women: The Sexual Satisfaction Scale for Women (SSS-W).Journal of Sexual Medicine, 2(1), 66-81.

Moyano, N., \& Sierra, J.C. (2012). Spanish adaptation and validation of the sexual cognitions checklist (SCC). Anales de Psicología 28(3), 904-914.

Muthén, L.K. and Muthén, B.O. (1998-2015). Mplus User's Guide. Seventh Edition. Los Angeles, CA: Muthén \& Muthén.

Öberg, K., Fugl-Meyer, K. S., \& Fugl-Meyer, A.R. (2002). On sexual wellbeing in sexually abused Swedish women: Epidemiological aspects. Sexual and Relationship Therapy, 17(4), 329-341.

Park, N., Peterson, C., \& Sun, J. (2013). La psicología positiva: Investigación y Aplicaciones. Terapia Psicológica, 31(1), 11-19.

Pascoal, P. M., Narciso, I., \& Pereira, N. M. (2014). What is sexual satisfaction? Thematic analysis of lay people's definitions. Journal of Sex Research, 51(1), 22-30. 
Programa de las Naciones Unidas para el Desarrollo (2012). Desarrollo humano en Chile 2012. Bienestar subjetivo: el desafío de repensar el desarrollo. Santiago de Chile.

R Development Core Team (2017). R: A language and environment for statistical computing. R Vienna, Austria: Foundation for Statistical Computing.

Revelle, W. (2017) psych: Procedures for Personality and Psychological Research. USA: Northwestern University, Evanston, Illinois,

Ribeiro, M.C., Nakamura, M.U., Scanavino, M.D., Torlonj, M.R. \& Mattsr R. (2011). Female Sexual Function and Gestional Diabetes. Journal of Sexual Medicine, 9(3), 786-792.

Rosen, R., \& Bachmann, G. (2008). Sexual well-being, happiness, and satisfaction in women. Journal of Sex \& Marital Therapy, 34(4), 291-297.

Rosen, R., Bachmann, G., Reese, J., Gentner, L., Leiblum, S., Wajszczuk, C., \& Wanser, R. (2009). Female Sexual Well-Being Scale (FSWB Scale): Developmental Psychometric Validation in Sexually Functional Women. The Journal of Sexual Medicine, 6(5), 1297-1305.

Sánchez-Fuentes, M., Salinas, J. M., \& Sierra, J. C. (2016). Use of an Ecological Model to Study Sexual Satisfaction in a Heterosexual Spanish Sample. Archives of Sexual Behavior, 45(8), 1973-1988.

Santos-Iglesias, P., \& Sierra, J.C. (2010). El papel de la asertividad sexual en la sexualidad humana: una revisión sistemática. International Journal of Clinical and Health Psychology, 10(3), 553-577.

Santos-Iglesias, P., Sierra, J.C., \& Vallejo- Medina, P. (2013). Predictors of Sexual Assertiveness: The Role of Sexual Desire, Arousal, Attitudes, and Partner Abuse. Archives of Sexual Behavior, 42(6), 1043-1052.

Sierra, J.C., Ortega, V., Martín-Ortiz, J.D., \& Vera-Villarroel P. (2004). Propiedades psicométricas del cuestionario de Wilson de fantasías sexuales. Revista Mexicana de Psicología, 21(1), 37-50.

Silva, P., Pereira, H., Esgalhado, G. Monteiro, S., Afonso, R.M., \& Loureir, M. (2016). Emotional Intelligence, Sexual Functioning, and Subjective Sexual Well-being in Portuguese Adults. British Journal of Education, Society \& Behavioural Science, 15(1), 1-11.

Stephenson, K. R., \& Meston, C. M. (2010a). Differentiating components of sexual well-being in women: Are sexual satisfaction and sexual distress independent constructs? Journal of Sexual Medicine, 7, 2458-2468.

Stulhofer, A., Busko, V., \& Brouillard, P. (2010). Development and bicultural validation of the new sexual satisfaction scale. Journal of Sex Research, 47(4), 257-68.

Træen, B., \& Schaller, S. (2010). Subjective Sexual Well-Being in a Web Sample of Heterosexual Norwegians. International Journal of Sexual Health, 22(3), 180-194.

Vallejo-Medina, P., \& Sierra J.C. (2013). Effect of drug use and influence of abstinence on sexual functioning in a Spanish male drug-dependent sample: A multisite study. Journal of Sexual Medicine, 10(2), 333-341.

Velten, J., Scholten, S., Graham, C. A., \& Margraf, J. (2017). Sexual excitation and sexual inhibition as predictors of sexual function in women: A cross-sectional and longitudinal study. Journal of Sex \& Marital Therapy, 43(2), 95-109.

Vera-Villarroel, P., Valtierra, A., \& Contreras, D. (2016). Affectivity as mediator of the relation between optimism and quality of life in men who have sex with men with HIV. International Journal of Clinical and Health Psychology, 16, 256-265.

Willi, J., \& Burri, A. (2015). Emotional Intelligence and Sexual Functioning in a Sample of Swiss Men and Women. The Journal of Sexual Medicine, 12(10), 2051-2060.

Woloski-Wruble, A., Oliel, Y., Leefsma, M., \& Hochner-Celnikier, D. (2010). Sexual activities, sexual and life satisfaction, and successful aging in women. Journal of Sexual Medicine, 7(7), 2401-2410.

World Health Organization (2010). Measuring sexual health: Conceptual and practical considerations and related indicators.

Zimmer-Gembeck, M. J., \& French, J. (2016). Associations of sexual subjectivity with global and sexual well-being: A new measure for young males and comparison to females. Archives of Sexual Behavior, 45(2), 315-327. 
